Nonlinear Processes in Geophysics (2005) 12: 117-128

SRef-ID: $1607-7946 / \mathrm{npg} / 2005-12-117$

European Geosciences Union

(c) 2005 Author(s). This work is licensed

under a Creative Commons License.

\title{
Nonlinear multidimensional scaling and visualization of earthquake clusters over space, time and feature space
}

\author{
W. Dzwinel ${ }^{1}$, D. A. Yuen ${ }^{2}$, K. Boryczko ${ }^{1}$, Y. Ben-Zion ${ }^{3}$, S. Yoshioka ${ }^{4}$, and T. Ito ${ }^{5}$ \\ ${ }^{1}$ AGH Institute of Computer Science, al. Mickiewicza 30, 30-059, Kraków, Poland \\ ${ }^{2}$ Minnesota Supercomputing Institute, Univ. of Minnesota, Minneapolis, MN 55455, USA \\ ${ }^{3}$ Department of Earth Sciences, University of Southern California, Los Angeles, CA 90089, USA \\ ${ }^{4}$ Department of Earth and Planetary Sciences, Kyushu University, Fukuoka, 812-8581, Japan \\ ${ }^{5}$ Graduate School of Environmental Studies, Nagoya, University, Furo-cho, Nagoya, Aichi, 464-8602, Japan
}

Received: 4 October 2004 - Revised: 1 December 2004 - Accepted: 2 December 2004 - Published: 28 January 2005

\begin{abstract}
We present a novel technique based on a multiresolutional clustering and nonlinear multi-dimensional scaling of earthquake patterns to investigate observed and synthetic seismic catalogs. The observed data represent seismic activities around the Japanese islands during 1997-2003. The synthetic data were generated by numerical simulations for various cases of a heterogeneous fault governed by 3-D elastic dislocation and power-law creep. At the highest resolution, we analyze the local cluster structures in the data space of seismic events for the two types of catalogs by using an agglomerative clustering algorithm. We demonstrate that small magnitude events produce local spatiotemporal patches delineating neighboring large events. Seismic events, quantized in space and time, generate the multidimensional feature space characterized by the earthquake parameters. Using a non-hierarchical clustering algorithm and nonlinear multi-dimensional scaling, we explore the multitudinous earthquakes by real-time 3-D visualization and inspection of the multivariate clusters. At the spatial resolutions characteristic of the earthquake parameters, all of the ongoing seismicity both before and after the largest events accumulates to a global structure consisting of a few separate clusters in the feature space. We show that by combining the results of clustering in both low and high resolution spaces, we can recognize precursory events more precisely and unravel vital information that cannot be discerned at a single resolution.
\end{abstract}

\section{Introduction}

Understanding of earthquake dynamics and development of forecasting algorithms require a sound knowledge and skill in both measurement and analysis spanning various geophysical data, such as seismic, electromagnetic, gravitational (Song and Simons, 2003), geodetic, geochemical, etc.

Correspondence to: D. A. Yuen

(davey@krissy.geo.umn.edu)
The Gutenberg-Richter power-law distribution of earthquake sizes (Gutenberg and Richter, 1944) implies that the largest events are surrounded (in space and time) by a large number of small events (e.g. Wesnousky, 1994; Ben-Zion and Rice, 1995; Wiemer and Wyss, 2002). The multi-dimensional and multi-resolutional structure of this global cluster depends strongly on geological and geophysical conditions (Miller et al., 1999; Ben-Zion and Lyakhovsky, 2002), past seismic activities (Rundle et al., 2000, 2002), closely associated events (e.g. volcano eruptions) and time sequence of the earthquakes forming isolated events, patches, swarms etc.

Investigations on earthquake predictions are based on the assumption that all of the regional factors can be filtered out and general information about the earthquake precursory patterns can be extracted (Geller et al., 1997). This extraction process is usually performed by using classical statistical or pattern recognition methodology. Feature extraction involves a pre-selection process of various statistical properties of data and generation of a set of seismicity parameters (Keilis-Borok and Kossobokov, 1990; Eneva and Ben-Zion, 1997a, b), which correspond to linearly independent coordinates in the feature space. The seismicity parameters in the form of time series can be analyzed by using various pattern recognition techniques ranging from fuzzy sets theory and expert systems (e.g. Wang and Gengfeng, 1996), multidimensional wavelets (Enescu et al., 2002; Erlebacher and Yuen, 2001, 2003) to neural networks (Joswig 1990; Dowla 1995; Tiira, 1999; Rundle et al., 2002; Anghel et al., 2004).

Prediction of earthquakes is a very difficult and challenging task (Geller et al., 1997); we cannot operate only at one level of resolution. The coarse graining of the original data can destroy the local dependences between the events and the isolated earthquakes by, e.g. neglecting their spatio-temporal localization. In coarse-grain analysis, the subtle correlations between the earthquakes and preceding patches of events can vanish in the background of uncorrelated and noisy data.

Multi-dimensional correlations can produce very distinct spatio-temporal patterns of seismic events. Tiampo et al. (2002a, b) provide recent evidence of event clustering in 


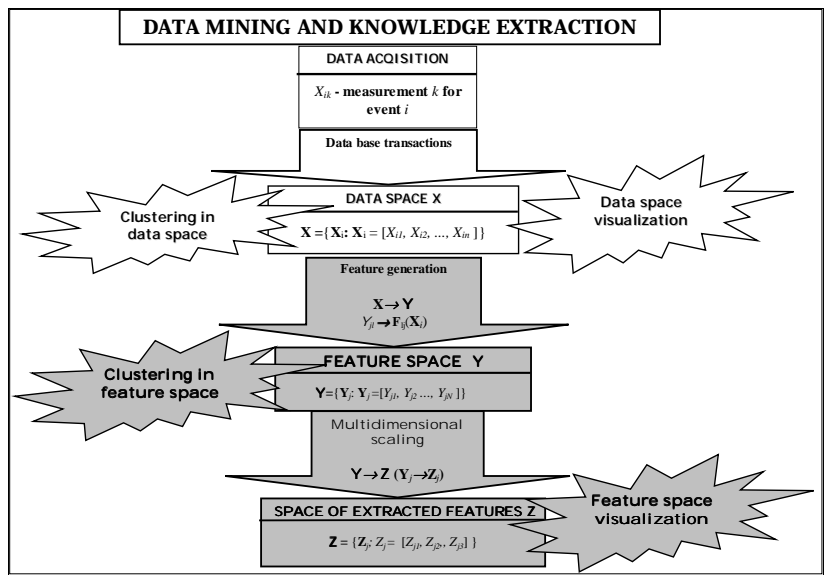

Fig. 1. The principal steps of the knowledge extraction process. The data space $X$ is represented by n-dimensional vectors $X_{i}$ of measurements $X_{k}$. It is transformed to a new abstract space $Y$ of vectors $\boldsymbol{Y}_{j}$. The coordinates $Y_{l}$ of these vectors represent seismic parameters, which are nonlinear functions of measurements $X_{k}$. The new features $Y_{l}$ form $N$-dimensional feature space. The multidimensional scaling procedure is used for visualizing the multidimensional events in 3-D space for a visual inspection of the $\mathrm{N}$ dimensional feature space.

seismicity catalogs. In Dzwinel et al. (2003), we proposed a new approach employing clustering for multivariate analysis of seismic data. The method can extract local spatiotemporal clusters of low magnitude events and recognize correlations between the clusters and the large earthquakes. We showed that these clusters could reflect clearly the short-term trends in seismic activities followed by isolated large events. However, local clustering of seismic events is not sufficient to extract an overall picture concerning the precursory patterns.

Our analysis procedure does not use a standard software package. Our goal is to construct an interactive system for data mining (Mitra and Acharya, 2003), which allows one to match the most appropriate clustering schemes for the structure of actual seismic data. As shown in Figs. 1 and 2, our data-mining techniques include not only various clustering algorithms but also feature extraction and visualization techniques. This present approach is more general than the work of (Dzwinel et al., 2003).

In this paper we propose a novel muti-resolutional approach, which combines local clustering techniques in the data space with a non-hierarchical clustering in the feature space. The raw data are represented by $n$-dimensional vectors $\boldsymbol{X}_{i}$ of measurements $X_{k}$. The data space can be searched for patterns and be visualized by using local or remote pattern recognition and advanced visualization capabilities. The data space $\boldsymbol{X}$ is transformed to a new abstract space $\boldsymbol{Y}$ of vectors $\boldsymbol{Y}_{j}$. The coordinates $Y_{l}$ of these vectors represent nonlinear functions of measurements $X_{k}$, which are averaged in space and time in given space-time windows. This transformation allows for coarse graining of data (data quantization),

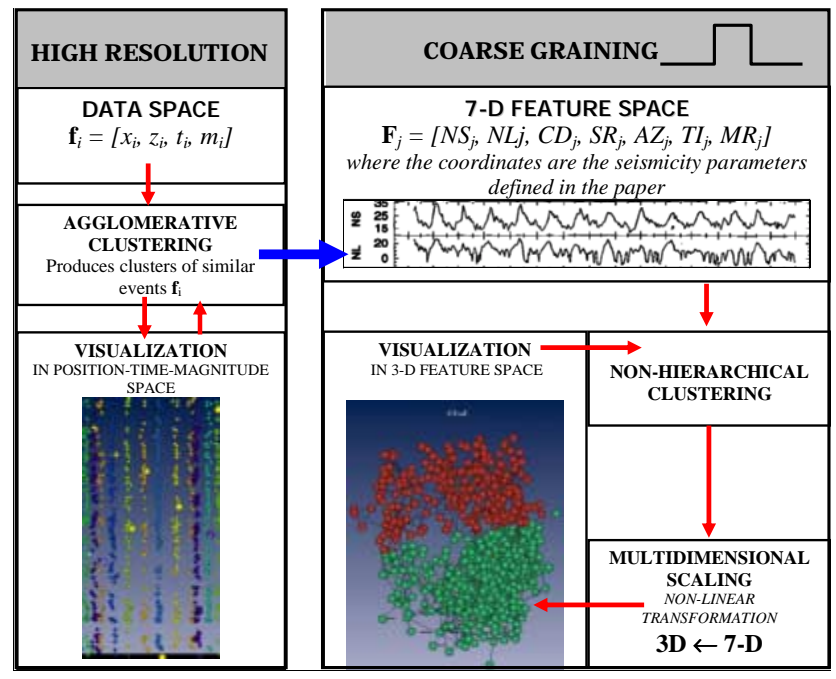

Fig. 2. Schematic diagram of multi-resolutional analysis of seismic events. At the highest level of resolution, a single seismic event $i$ is represented as a multi-dimensional data vector $f_{i}$. These vectors contain information about local properties of seismic patterns. A general knowledge about the data has to be extracted from the lower resolution feature space $\Omega$ by using coarse graining procedure $L[\Phi]$. The MDS transformation $M S[\Omega] \rightarrow \omega$ maps the 7-D feature space $\Omega$ into its image in a 3-D space $\omega$.

amplification of their characteristic features, and suppression of both the noise and other random components. The new features $Y_{l}$ form an $N$-dimensional feature space. We use multi-dimensional scaling procedures for visualizing the multi-dimensional events in 3-D space. The Sammon's nonlinear transformation (Jain and Dubes, 1988) (multidimensional scaling), transforms $\boldsymbol{Y}$ into a 3-D $\mathbf{Z}$ space of extracted features, assuming that this dimensionality reduction minimizes the distortions between the $N$-dimensional structure of $\mathbf{Y}_{j}$ vectors and its 3-D image in $\mathbf{Z}$ space. This transformation allows for a visual inspection of the $N$-dimensional feature space. The visual analysis helps greatly in detecting subtle cluster structures, not recognized by classical clustering techniques, selecting the best pattern detection procedure used for data clustering, classifying the anonymous data and formulating new hypotheses.

We used our methodology for analyzing the observed (Ito and Yoshioka, 2002; Toda et al., 2002) and synthetic (BenZion, 1996) earthquake data. The observed data represent seismic activity of the Japanese islands in the 1997-2003 time interval. The synthetic catalogs correspond to various cases of a large heterogeneous fault zone in an elastic halfspace.

The reminder of the paper is constructed as follows. First, we provide background material on multi-resolutional and visual clustering and data-mining. Then we describe the clustering and multi-dimensional scaling methods we use for recognition of seismic anomalies. This is followed by results of data analysis both for the observed and the synthetic seismic catalogs. We show that the multi-resolutional ap- 
proach can indeed improve greatly the accuracy of following the evolution of earthquake dynamics. Finally, we discuss the conclusions and future prospects.

\section{Methodologies}

2.1 Multi-resolutional analysis of multidimensional seismic data

In Fig. 2, we show that the seismic data can be analyzed in diverse resolutions, associated with two different types of spaces:

1. The first is the data space $\Phi$ with data vectors $f_{i}$ $(\mathrm{i}=1, \ldots, N)$ - which correspond to the data describing a single seismic event.

2. The second one, more abstract, is the feature space $\Omega$ of time events $\boldsymbol{F}_{j}(\mathrm{j}=1, \ldots, M)$ and $M \ll N-$ an abstract space resulting from a non-linear transformation $\boldsymbol{L}[\Phi] \rightarrow \Omega$ representing the feature generation procedure.

At the highest level of resolution, a single seismic event $i$ can be represented as a multi-dimensional data vector $\boldsymbol{f}_{i}=\left[m_{i}\right.$, $\left.z_{i}, x_{i}, t_{i}\right]$ where $m_{i}$ is the magnitude and $x_{i}, z_{i}, t_{i}$ - its epicentral coordinates, depth and the time of occurrence, respectively. As shown in (Dzwinel et al., 2003), we can analyze these data locally by looking for clusters with similar (or dissimilar) events using the agglomerative clustering procedures (Andenberg, 1973; Gowda and Krishna, 1978; Jain and Dubes, 1988; Theodoris and Koutroumbas, 1998). The search for similar data is limited to successive time stripes $t_{k}$ with the same width $\Delta \tau$. We are seeking neighbors of event $i$ only in $t_{k}$ and the previous $t_{k-1}$ time intervals. The point $j$ belongs to the nearest neighbors of event $i$ if a given set of conditions is fulfilled (see Dzwinel et al., 2003). This allows us to identify correlated patches of events that reflect shortterm trends in seismic activity initiated by rapid changes generated by strong events. By combining similarity and dissimilarity measures (Theodoris and Koutroumbas, 1998) between the data vectors, we can extract also the patches of small magnitude events corresponding to the isolated large earthquakes. This type of data analysis extracts information on the local properties of seismic patterns (Dzwinel et al., 2003). However, this also generates a large number of extraneous clusters, which produce unreliable information over a long timescale.

With the above procedure, we cannot extract general knowledge about the data, which requires the detection of long-range spatial and temporal correlations. This knowledge has to be extracted from a global data structure in a low resolution space $\Omega$. We achieve this by using a coarse graining procedure $\boldsymbol{L}[\Phi]$. This averages out the noise and detailed modes of the data vector components.

The coordinates in the low resolution feature space are defined by means of seismicity parameters. Originally they are computed as time $\tau$ and space $X$ averages in a given time $\left[t_{0}, t_{\mathrm{END}}\right]$ and space intervals $\left(\left[\boldsymbol{X}_{0}, \boldsymbol{X}_{\mathrm{END}}\right]=\left[x_{0}, x_{\mathrm{END}}\right] \cup\right.$ $\left[z_{0}, z_{\mathrm{END}}\right]$ - epicentral coordinates and depth, respectively) within a sliding time window with a length $\Delta T$ and time step $d t$, i.e.

$\alpha_{i}=\int_{t_{0}}^{t_{\mathrm{END}}} \int_{\boldsymbol{X} \in\left[\boldsymbol{X}_{0}, \boldsymbol{X}_{\mathrm{END}}\right]} \alpha(\tau, X) \cdot H\left(t_{0}+i \cdot d t, \tau\right) d \tau d X$

$H(t, \tau)=\left\{\begin{array}{c}1 \text { for }-\frac{\Delta T}{2}<\tau-t<\frac{\Delta T}{2} \\ 0 \text { otherwise }\end{array}\right.$

where $\alpha$ represents one of the following seismicity parameters: $N S, N L, C D, S R, A Z, T I, M R$. The value of $d t$ was assumed to be equal to the average time difference between two recorded consecutive events while $\Delta T$ is equal to about $1 / 10$ of the average time distance between two successive large events (for synthetic data $m>6$, for real data $m>5$ ). Larger values of $d t$ and $\Delta T$ give smoother time series due to better statistics. However, by increasing $d t$ and $\Delta T$ poorer prediction characteristics can be expected. The seismicity parameters are defined as follows (Eneva and BenZion, 1997a, b):

Degree of spatial non-randomness at short $(N S)$ and at long distances $(N L)$ - represents the differences between distributions of event distances and distances between randomly distributed points. NS and NL represent the portions of events involved in anomalies in short distances and long distances, respectively $(0-5 \mathrm{~km}$ and $60-65 \mathrm{~km}$ for the synthetic catalogs, Eneva and Ben-Zion, 1997).

1. Spatial correlation dimension $(C D)$ - calculated on the basis of correlation integrals based on interevent distances.

2. Degree of spatial repetitiveness $(S R)$ - contains the spatio-magnitude. components and represents the tendency of events with similar magnitudes to have nearly the same locations of hypocenters.

3. Average depth $(A Z)$.

4. Inverse of seismicity rate $(T I)$ - time interval in which a given (constant) number of events occurs.

5. Ratio of the numbers of events falling into two different magnitude ranges $M R=N\left(m \geq M_{0}\right) / N\left(m<M_{0}\right)$.

We have introduced an additional parameter $M$, which is not used in the data processing and simply displays the maximum magnitude of events in the moving time window.

We focus our data analysis on the time series of seven seismicity parameters that create the abstract 7-dimensional feature space of time events $F_{t}=\left(N S_{t}, N L_{t}, C D_{t}, S R_{t}, A Z_{t}\right.$, $T I_{t}, M R_{t}$ ) where $t$ are discretized values of time. These events produce clusters, which correspond to similar (or dissimilar) fragments of a 7-dimensional time series. Thus the clusters have information about the anomalies reflected by 
all the 7 parameters in the same moment of time. Because the number of clusters is generally unknown, and most of the clustering methods are not able to extract the clusters of complicated shapes and densities accurately (Ertoz et al., 2003), we propose to visualize the clustering structure in the feature space. We use multi-dimensional scaling transformation $M S$ $[\Omega] \rightarrow \omega$, which maps the 7-D feature space $\Omega$ into its image in a 3-D space $\omega$ (Jain and Dubes 1988; Siedlecki et al., 1988; Theodoris and Koutroumbas, 1998; Dzwinel, 1994; Dzwinel and Blasiak, 1999). From high-resolution 3-D visualization, one can discern clearly how strong the clusters are and how they are positioned with respect to each other. This allows us to fine tune the clustering parameters or select a different clustering algorithm that matches better the clustered structures.

\subsection{Clustering schemes}

Clustering analysis is a mathematical concept whose main useful role is to extract the most similar (or dissimilar) separated sets of objects according to a given similarity (or dissimilarity) measure (Andenberg, 1973). This concept has been used for many years in pattern recognition. Nowadays clustering and other feature extraction algorithms are recognized as important tools for revealing coherent features in the earth sciences (Rundle et al., 1997, 2000; Freed and Lin, 2001), bioinformatics (Jones and Pevzner, 2004) and in data mining (Xiaowei et al., 1999; Grossman et al., 2001; Hand et al., 2001; Hastie et al., 2001; Mitra and Acharya, 2003). Depending on the data structures and goals of classification, different clustering schemes must be applied (Gowda and $\mathrm{Kr}$ ishna, 1978; Karypis and Kumar, 1999).

In our new approach we use two different classes of clustering algorithms for different resolution levels. In data space we use agglomerative schemes, such as modified mutual nearest neighbor algorithm (mnn) (Gowda and Krishna, 1978; Karypis et al., 1999; Boryczko et al., 2003). This type of clustering extracts better the localized clusters in the highresolution data space.

In the feature space we are searching for global clusters of time events comprising similar events from the whole time interval. The non-hierarchical clustering algorithms are used mainly for extracting compact clusters by using global knowledge about the data structure. We use improved kmeans based schemes (Theodoris and Koutroumbas, 1998), such as a suite of moving schemes (Ismail and Kamel, 1989), which uses the k-means procedure plus four strategies of its tuning by moving the data vectors between $\mathrm{k}$-clusters to obtain a more precise location of the minimum of the goal function:

$J(\mathbf{Z})=\sum_{j} \sum_{i \in C_{j}}\left|\mathbf{x}_{i}-\mathbf{z}_{j}\right|^{2}$,

where $\mathbf{Z}=\left[\mathbf{z}_{1}, \ldots, \mathbf{z}_{k}\right], \mathbf{z}_{j}$ is the position of the center of mass of the cluster $j$, while $\mathbf{x}_{i}$ are the feature vectors closest to $\mathbf{z}_{j}$. To find a global minimum of function $J()$, we repeat many times the clustering procedures for different initial condi- tions. Each new initial configuration is constructed in a special way from the previous results by using the methods from (Ismail and Kamel, 1989; Zhang and Boyle, 1991). The cluster structure with the lowest $J(w, z)$ minimum is selected.

\subsection{Multidimensional scaling (MDS)}

The feature extraction methods, called also mapping techniques or multi-dimensional scaling (MDS), represent linear or non-linear transformations of $\mathrm{N}$-dimensional data into $n$-dimensional sets, where $n \ll N$ (Jain and Dubes, 1988; Siedlecki et al., 1988; Theodoris and Koutroumbas 1998; Dzwinel, 1994; Dzwinel and Blasiak, 1999). These methods allow for visualization of the multidimensional data in 3-D and for participating interactively the process of cluster extraction.

The MDS algorithm, which is based on the "stress function" criterion, is one of the most powerful mapping techniques. The goal is to maintain all the distances between points $R_{i} \in \omega \subset \mathfrak{R}^{N}$ in the Euclidean 3-D (or 2-D) space with a minimum error. The "stress function" criterion is as follows:

$E\left(\omega, \omega^{\prime}\right)=\sum_{j<i} D_{i j}^{-w m} \cdot\left(D_{i j}-r_{i j}^{\prime}\right)^{d}=\min$

where:

$r_{i j}^{\prime}=\left(\mathbf{r}_{i}-\mathbf{r}_{j}\right) \cdot\left(\mathbf{r}_{i}-\mathbf{r}_{j}\right), \quad i, j=1, \ldots, M$.

$D_{i, j}$ - is a squared distances between points $\mathbf{R}_{i}, \mathbf{R}_{j} \in \omega \subset \mathfrak{R}^{N}$ and $\mathbf{r}_{i}, \mathbf{r}_{j} \in \omega^{\prime} \subset E^{3}$ - coordinates of the respective points in 3 -D Euclidean space and $w, d$-parameters.

The result of mapping depends on the quality of the minimum obtained for the criterion function (Eq. 4). The dimensionality of the "stress function" domain is very high and is equal to $N \cdot M$ (thousands, in typical problems). An increase of the number of input data (more than $10^{3}$ ), the dimensionality of source space and data complexity may cause the resulting 2-D (3-D) patterns to be completely illegible. This is often the case with application of standard numerical algorithms for finding minimum of this multimodal, non-linear and complex criterion. To make the non-linear mapping useful for visualization of greater $\left(M>10^{3}\right.$ and $\left.N>10^{2}\right)$ data samples, a new minimization technique extracting global minimum of the criterion function is required.

We propose to use the molecular dynamics algorithm (Dzwinel and Blasiak, 1999) as a solver, which can be used for finding the global minimum of the criterion function (Eq. 4). Let us assume that:

1. an initial configuration of $M$ mutually interacting "particles" is generated in $E^{3}$,

2. every "particle" corresponds to the respective $N$ dimensional point from $\mathfrak{R}^{N}$,

3. the "particles" interact with each other with $\Phi_{i, j}$ particle-particle potential where 

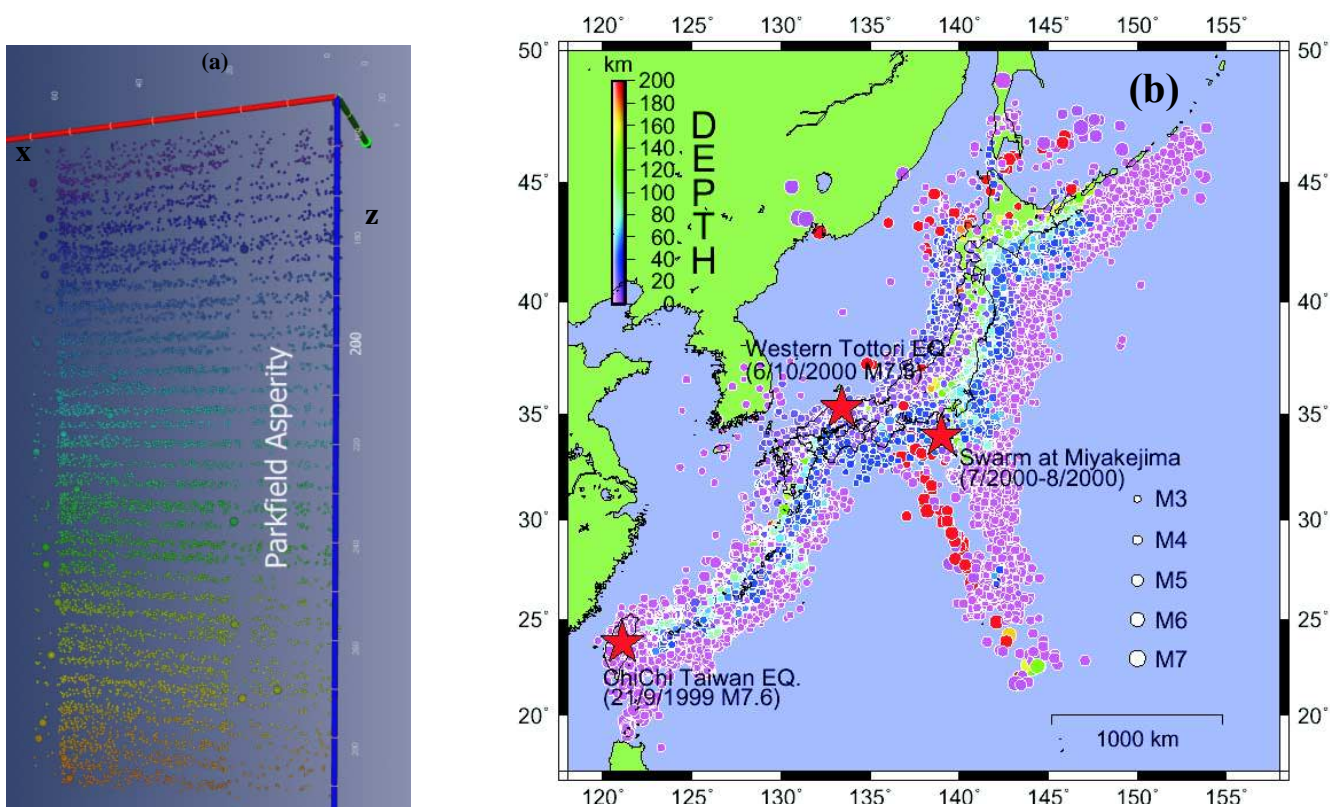

Fig. 3. (a) The synthetic raw data (horizontal distance $-X$, depth $-z$ ) visualized in time by using the Amira visualization package (http: //www.amiravis.com) for A data set (Ben-Zion, 1996). Large events $(m>6)$ are shown as distinctly larger dots on the background of low magnitude events $(m<4)$. There are visualized patches of low magnitude events preceding larger events (Dzwinel et al., 2003). The patches represent clusters marked in colors. (b) Seismic activities around the Japanese Archipelago within 5 years time period. We use the hypocentral data provided by the Japan Meteorological Agency (JMA). The magnitude of the earthquakes (JMA magnitude) and their depths are represented by differences of the radius of the circle and colors, respectively. The red stars symbolize large events such as: Chi-Chi Taiwan earthquake (21 September 1999 M7.6 latitude 23.8 longitude 121.1) Swarm at Miyakejima (July 2000- August 2000 latitude 34.0 longitude 139.0) Western Tottori earthquake (6 October 2000 M7.3 latitude 35.3 longitude 133.4).

$\Phi_{i, j}=\frac{k}{2 m} D_{i j}^{-w m} \cdot\left(D_{i j}-r_{i j}^{\prime}\right)^{d}$

and $k$ is a stiffness factor. Thus the interaction between each pair of particles is described by various long range potentials, dependent on the separation distance between particles $r_{i j}$ and the distance $D_{i j}$ between respective multidmensional points in $\Re^{N}$. To assure the energy dissipation from the system, an additional friction force is introduced. Using the "leap-frog" numerical scheme (Haile, 1992) the following formula for velocities and positions of "particles" can be derived from the momentum equation:

$\mathbf{v}_{i}^{n+1 / 2}=\frac{(1-\varphi)}{(1+\varphi)} \cdot \mathbf{v}_{i}^{n-1 / 2}+\frac{\alpha \Delta t}{(1+\varphi)} \cdot\left\{\sum_{j=1}^{M}\left(r_{i j}^{n}-D_{i j}\right)^{d-1} \mathbf{r}_{i j}^{n}\right\}$,

$\mathbf{r}_{i}^{n+1}=\mathbf{r}_{i}^{n}+\mathbf{v}_{i}^{n+1 / 2} \cdot \Delta t$,

$\alpha=\frac{k}{m}, \quad \varphi=\frac{\lambda}{2 m} \cdot \Delta t$,

where $\mathbf{v}_{i}^{n}, \mathbf{r}_{i}^{n}$ - the velocity and position of particle $i$, respectively, $n$ - time-step number, $m=1$ - particle mass.

As it is in molecular dynamics (Haile, 1992), the system of "particles" described by the discrete Eqs. (6) evolves in time according to the Newton equations of motion until the global (or close to the global) minimum of Eq. (5) is reached. Only two free parameters, $\lambda$ and $k$, have to be fit to obtain the proper stable state, where the final positions of frozen "particles" reflect the result of N-D to 3-D mapping.

\section{Description of the data}

We analyze the observed and synthetic earthquake catalogs for different time intervals. The synthetic catalogs (Fig. 3a) were obtained by numerical simulations of seismicity on a heterogeneous fault governed by 3-D elastic dislocation theory, power-law creep and boundary conditions corresponding to the central San Andreas Fault (Ben-Zion, 1996).

The synthetic seismicity described in (Ben-Zion, 1996; Eneva and Ben-Zion, 1997) is distributed in space (horizontal distance $X$, depth $z$ ), time $t$ and magnitude $m$ size. A large 1857-type event is imposed at the south in the beginning of the simulation and a large 1906-type event is imposed at the north 50 years later. We study catalogs from four different model realizations, representing various levels of fault zone heterogeneities. These are models with statistically uniform brittle properties (U), with a Parkfield type Asperity (A), with fractal brittle properties (F), and with multi-sizeheterogeneities (M). The basic characteristics of the data are given in Table 1. These models and various statistical properties of the catalogues have been discussed in greater detail elsewhere (Ben-Zion, 1996; Eneva and Ben-Zion, 1997). The examined time interval covers every event, which occurred during the last 150 years of the simulated fault activity and this period contains $1-3 \times 10^{4}$ events in the magnitude interval (3.3-6.8). The seismicity parameters were obtained by averaging the data using a sliding time window of constant 
Table 1. Data specification.

\begin{tabular}{ccccc}
\hline Catalog & Total number of events & Time interval (years) & Events with $m>6$ & Earthquake magnitude \\
\hline JMA data & 42370 & 5 & 62 & $3<m<7.90$ \\
U & 32185 & 150 & 32 & $3.26<m<6.68$ \\
A & 25881 & 150 & 30 & $3.26<m<6.73$ \\
F & 10475 & 150 & 16 & $3.43<m<6.73$ \\
M & 29039 & 150 & 20 & $3.41<m<6.81$ \\
\hline
\end{tabular}

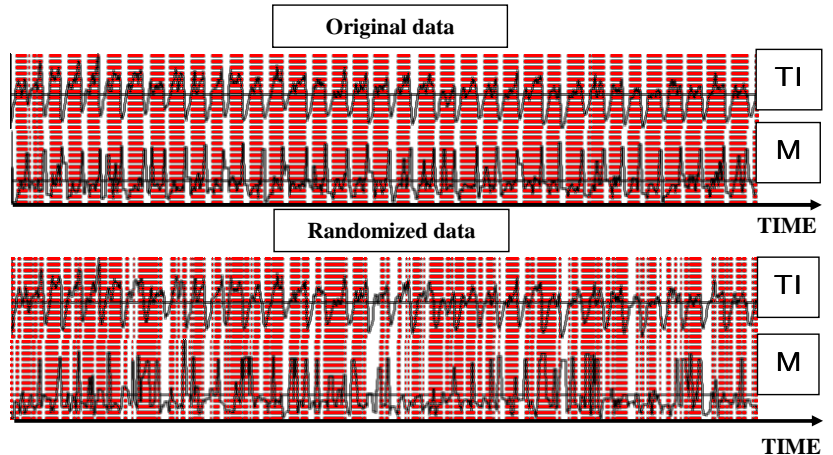

Fig. 4. The seismicity parameters $T I$ and $M$ for the "original" and "randomized" synthetic data sets (set A). There is not any correlations between $M$ and $T I$ for the randomize data. This is a contrast to the original data set.

width $\Delta T$ and shift $d t$ (see Eqs. 1-2). We employ $\Delta T=10$ days and $d t=2$ days for the Japanese data and $\Delta T=10$ months and $d t=2$ months for the synthetic data. Each parameter in the clustering was normalized with respect to the standard deviation.

The observed data (see Fig. 3b) represents seismic activities of the Japanese islands collected by the Japan Meteorological Agency (JMA). The JMA catalogue consists of 915829 events detected in Japan Islands between 1923 and 31 January 2003. The original catalogue includes also events with magnitudes less then 1.0. The lowest magnitudes were determined by using a detection level, estimated from the Gutenberg-Richter frequency-size distribution. For the purposes of this paper we have assumed that the cutoff magnitude of earthquake is equal to $3(m>3)$. We do not use any cutoff depth of hypocenter events. The seismic events shown in Fig. 3b, were recorded during the 5 years time interval from 1 October 1997 to 31 January 2003. The data set processed consists of 42370 seismic events with magnitudes $m$, position in space (latitude $X$, longitude $Y$, depth $z$ ) and occurrence time $t$. Statistical completeness of the earthquakes above the detection level assures that no significant events in both space and time are missing.

\section{Results of clustering}

As shown in Fig. 3a and in (Dzwinel et al., 2003), the synthetic seismic events with magnitudes $m<4$ produce stripe- like clusters in the data space. They precede large earthquakes $(m>6)$ and are separated in time by the regions of quiescence. Similar pattern can be observed in the feature space.

In Fig. 4 we display the time series for two, out of seven, seismicity parameters: the maximum magnitude of events in the moving time window $M$ and the inverse seismicity rate $T I$, which define the time distribution of events. The seismicity parameters were computed both for the raw data set A from the synthetic data catalog ("original data") and for the same data set but randomized in time ("randomized data"). These two sets of data were searched for clusters in the 7$D$ feature space $\Omega$. For simplicity, only two clusters were considered. They are represented in Fig. 4 by red and white stripes.

As shown in Fig. 4, the $M$ and $T I$ time series from the "original data" are highly correlated. The periodic occurrence of large events with $m>6$ is preceded by the increase of the inverse of seismicity rate $T I$ (the region of quiescence), which drops at the large event time. Similar correlations can be seen for other seismicity parameters not displayed in Fig. 4. The time intervals corresponding to these rapid changes of seismicity parameters produce one cluster (white), while the rest of them belong to the second cluster (red). Moreover, the periodic stripes representing time intervals from the second cluster correspond to the clusters of events in the data space displayed in Fig. 3a. This shows that the averaged properties of a variety of clusters from the data space (see Fig. 3a) are similar, producing a single supercluster in the feature space.

It is obvious that clusters can be also found for the "randomized data", however, they are completely meaningless i.e. computed in-cluster average correlations are statistically irrelevant. This can be inspected visually in Fig. 4. The correlations between seismicity parameters - including the correlation between the inverse of seismicity rate $T I$ and the averaged magnitude $M$ - do not exist for the "randomized data". It is also impossible to extract any distinct properties separating the two clusters (e.g. by using the KarhunenLoeve transformation, see e.g. Theodoris and Koutroumbas, 1998).

By comparing these results of clustering for original and randomized data sets, we can conclude that seismic events create informative causal patterns, which can be extracted both in the data and feature spaces by using local and global 
clustering schemes, respectively. These patterns contain information concerning the correlations between characteristic features of seismic measurements and their temporal sequencing. This can be confirmed further by additional analysis of both the synthetic and observed seismic data.

In Fig. 5 we display the seismicity parameters in time computed for the complete synthetic data catalog A. The time events $\boldsymbol{F}_{t}=\left(N S_{t}, N L_{t}, C D_{t}, S R_{t}, A Z_{t}, T I_{t}, M R_{t}\right)$ produce 3 clusters in the feature space: the first cluster symbolized by green, the second by white and the third by red strips. From the top plot of Fig. 5 displaying the largest events $M$ in the sliding time window, we may conclude that the white and red clusters comprise time events $\boldsymbol{F}_{t}$, which correspond to post mainshock effects. The white cluster represents the net aftershock events while the red one includes the earthquake effects averaged in the sliding time window. Conversely, the green cluster contains the time events $\boldsymbol{F}_{t}$ preceding the earthquakes. The selectivity in time of the seismicity parameters depends on the width $\Delta T$ and shift $d t$ of the sliding time window. Due to space and time averaging, it is impossible to correlate precisely the appearance of a given earthquake with the rest of the seismicity parameters when two earthquakes are too close to each other. Therefore, the sequence of green-red-white cluster events can be broken for the time domains with many large earthquakes (see Fig. 5). As shown in Fig. 5, the occurrence of the largest events correlates well with the minima of $N S, C D, S R, T I$ and maxima of $A Z, M R$ parameters. This means that the occurrence of large earthquakes is preceded by increasing spatial diffusion of events and increasing inverse of seismicity rate. More specifically, the results reveal:

1. an increasing spatial randomness of seismic events,

2. a high spatial correlation dimension (this drops rapidly at the onset of large events),

3. a decreasing tendency of events with similar magnitudes to have nearly the same locations of hypocenters,

4. a decreasing average depth of seismic activity,

5. an increase of inverse seismicity rate before large events (it drops rapidly at the onset of large event).

For the synthetic data the clusters in the feature space reflect well both the precursory and post mainshock effects. However, the interpretation of clusters for the real data is more complicated and ambiguous.

Results of clustering of the observed Japanese seismic catalogs both in data and in feature spaces are shown in Fig. 6 . At the highest resolution level a single seismic event $i$ can be represented as a multi-dimensional data vector $\boldsymbol{f}_{i}=\left[m_{i}, z_{i}\right.$, $X_{i}, Y_{i}, t_{i}$ ] where: $m_{i}$ is the magnitude, $X_{i}$ - the latitude, $Y_{i}$ - the longitude, $z_{i}$ and $t_{i}$ - the depth and the time of occurrence, respectively. The seismic events are visualized with the Amira visualization package (http://www.amiravis.com) in Figs. 5a and 5b as an irregular cloud consisted of colored dots with $(z, x, t)$ coordinates.

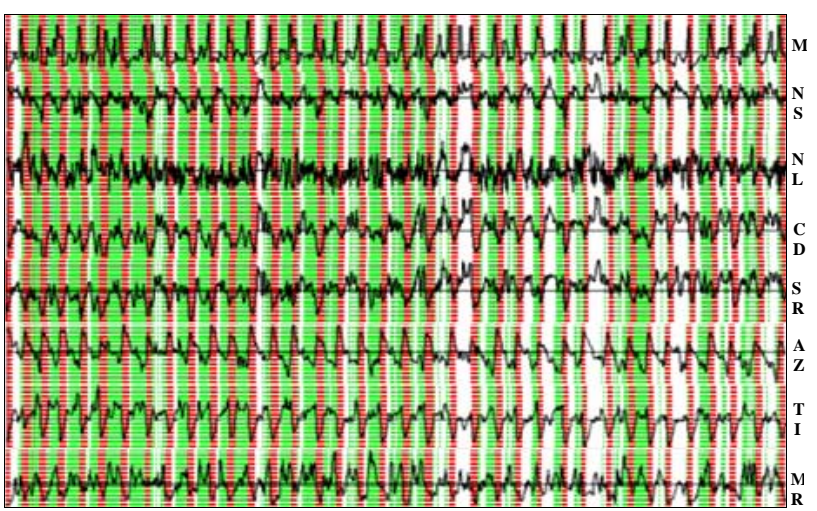

Fig. 5. The seismicity parameters $M, N S, N L, C D, S R, A Z, T I$, $M R$ in time (see Eqs. 1-2) for synthetic data catalog A.

From the Gutenberg-Richter relationship, the number of events of different range of magnitudes differs considerably. Therefore, we divide the entire set of data into three subsets comprising the small $S\left(m_{i}<m a\right)$, medium $M\left(m a \leq m_{i}<\max \right)$ and the large magnitude events $L\left(m_{i}>\max \right)$. The last ones represent the earthquakes and are displayed in Figs. 5a and 5b as larger spheres. The deepest earthquakes $z>150 \mathrm{~km}$ are not displayed in the Fig. 5 . The various shades represent the magnitudes of earthquakes from $m=6$ (green) to $m=7$ (red). In Figs. 5a and 5b we present the clustering results in the data space $\Phi$ of the data vectors $\boldsymbol{f}_{i} \in S\left(m_{i}<m a\right)$ (Fig. 5a) and $\boldsymbol{f}_{i} \in M\left(m a \leq m_{i}<\max \right)$ (Fig. $5 b)$. We have chosen arbitrarily that $m a=4$ and $\max =6$. We look for clusters of similar events as shown in (Dzwinel et al., 2003). The dots (data vectors), belonging to the same clusters, have the same color.

As shown in the upper part of Fig. 6a, clusters made of small size events are located mainly close to the surface $(0-$ $30 \mathrm{~km}$ deep). They form long disparate stripes along the time axis. The stripes break-out close to the largest cluster of earthquakes - the Miyakejima event (Toda et al., 2002; Ito and Yoshioka, 2002) - located in the middle of time interval and encircled in red in Fig. 6a. The large swarm of earthquakes (26 June 2000) occurs in the region of Miyakejima, Honshu, in central Japan (Toda et al., 2002; Ito and Yoshioka, 2002). The eruptions of Miyakejima and five large earthquakes with magnitudes 6.0 and above occurred together with a large number of 100000 smaller earthquakes.

Many other compact and small clusters are strongly correlated with this cluster. The second patch of large events encircled in white in Figs. $6 \mathrm{a}$ and $6 \mathrm{~b}$ - representing large swarm of earthquakes in the northern part of Japan (see Fig. 2a) is shown on the left hand side of the largest one. One can see clearly two clusters (encircled in blue in Fig. 6a) of similar depth preceding these two largest patches of earthquakes. Other clusters, such as the wide one spanned by a blue cluster and another smaller one, are located much deeper (larger than $100 \mathrm{~km}$ ) and represent the seismic background for the earthquakes occurring at depth up to $200 \mathrm{~km}$. Clusters of the 

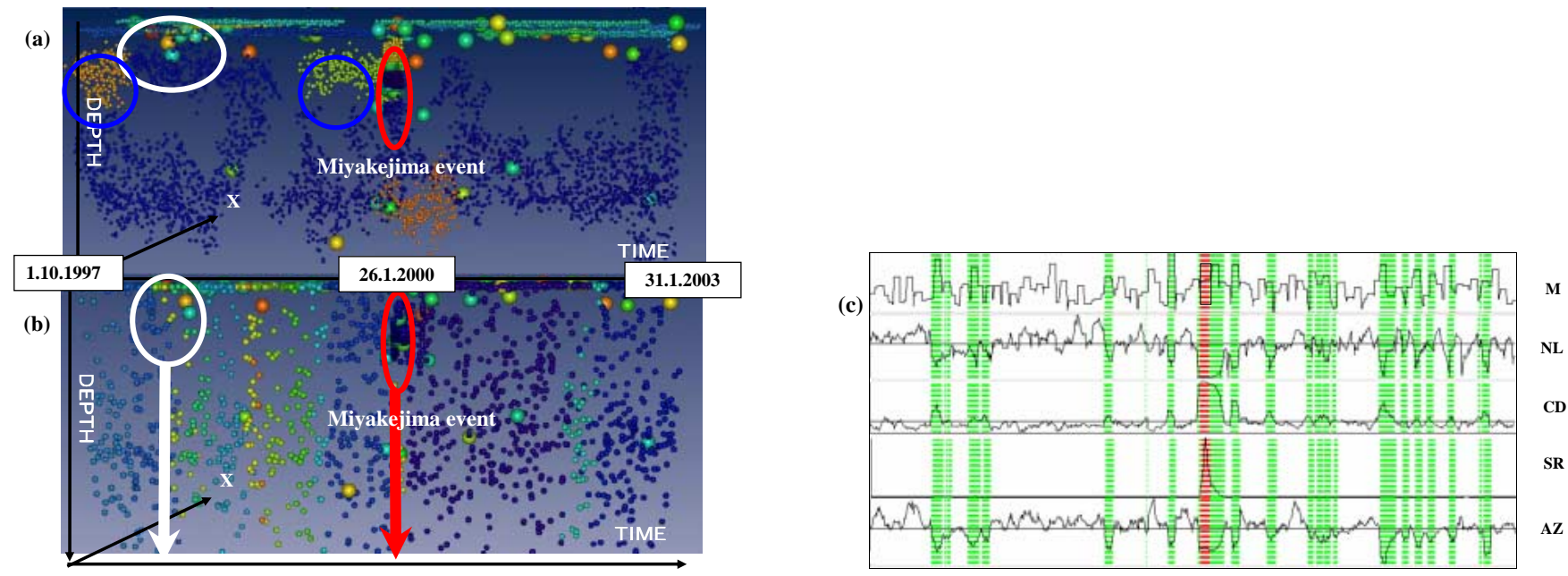

Fig. 6. Natural seismic data (Ito and Yoshioka, 2002) analyzed by using multi-resolutional clustering in both the data and the feature spaces (a)-(c). In panels a and b one can see the results of clustering in the data space for small magnitude $(3<m<4)$ and medium magnitude $(4<m<6)$ events, respectively, represented by the small shaded dots. The different colors of the dots mean different clusters. Large events are visualized by the larger spheres. The shades show difference in magnitudes $m$ (red - the largest, green - the smallest). The clusters in panels a-b encircled in red and white shows the places of the largest seismic activity, while those in blue probably represent the clusters of precursory events. The red, white and green stripes in panel c representing 4 (out of 7) seismic parameters and maximum magnitude $M$ show the time events belonging to three different clusters. The earthquakes are visualized by using the 3-D Amira visualization package (http://www.amiravis.com).

medium events $(4<m<6)$ (Fig. 6b) have completely different structures. They look like stripes, which lie parallel to $\mathrm{X}-\mathrm{Z}$ plane. The borders between clusters roughly correspond to the borders of successive showers of the earthquakes.

By clustering the time events in the feature space and simultaneous inspection of the results by using multidimensional scaling, we have found 4 distinct clusters. The cluster structure in 7-D feature space is shown in Fig. 7a, which represents the result of its mapping into 3-D. The blue cluster forming a long thin rod in Fig. 7a, corresponds to the famous Miyakejima earthquake swarm (Toda et al., 2002) encircled in red on Figs. 6a and 6b. The red and flat cluster from Fig. 7a, represent both the largest earthquakes and corresponding time events. The yellow and blue clusters contain the rest of the time events. The small blue cluster from Fig. 6a represents the events at the end of the time interval, which are averaged within a shrinking time window. In summary, clustering of averaged time events in the feature space does not detect any anomalies reflecting the precursory patterns.

In Fig. 6c we display the time series of selected seismicity parameters and in Fig. 7b the 3-D image of the feature space. Both pictures represent the data pre-selected initially by clustering the raw seismic events in the data space. Only small and medium events belonging to the largest clusters (displayed in Figs. 6a and $6 \mathrm{~b}$ ) were used for computing the seismicity parameters. Neglecting the events that do not produce clusters in the data space, we reduce the number of uncorrelated events both in space and in time enhancing extremes in the time series of seismicity parameters. As shown in Figs. $7 \mathrm{a}$ and $7 \mathrm{~b}$, in comparison to the original data, the feature space becomes more diverse producing several well separated clusters. In Fig. 6c, we show that by clustering data in the feature space, we can extract not only Miyakejima event (red cluster in the center) but also the cluster of events which are characterized by similar behavior of the seismicity parameters as the Miyakejima swarm (green cluster). The remaining data produce the white cluster. As shown in Fig. 6c, the green cluster comprises time events corresponding mainly to the largest and shallow earthquakes, which are characterized by increasing randomness of event locations, low spatial correlation dimension which increases rapidly in the moment of large shock, high spatial repetitiveness and high seismicity rate. The last properties are opposite to those observed for the synthetic catalog A. The differences are caused by the association of the two data sets with very different seismic regimes. Comparing the properties of red, green and white clusters we can conclude that the large swarm in the observed data is preceded and followed by events of a similar nature but considerably smaller magnitude. We note that the number of aftershocks is greater than the number of precursory effects belonging to the same green cluster.

The above results cannot be used yet for earthquake prediction. It is impossible to forecast earthquakes from just a single case. However, continuing analysis of this type may help to find "dangerous" seismic patterns and predict salient aspects of their evolution.

The prediction of earthquakes is simpler for the synthetic data, due to the simpler associated fault system (a single large strike-slip fault), the completeness of the data and the time interval covering many large earthquake cycles. Because the 


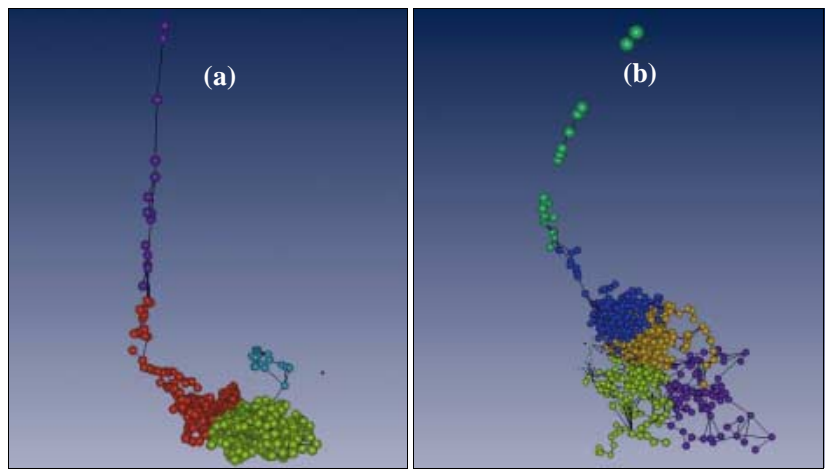

Fig. 7. The results of non-linear multi-dimensional scaling of 7-D clusters from the feature spaces defined by the seismicity parameters computed for all the seismic events from the data space to 3-D metric space (a) and the same data pre-selected initially by clustering (b). The figures are rendered using the Amira visualization package (http://www.amiravis.com).

precursory events produce distinct clusters in both Fig. 4 and Fig. 5, we can construct a simple visual classifier (Jain and Dubes 1988; Theodoris and Koutroumbas 1998) for recognizing the precursory patterns.

The entire time interval in the feature space has been divided into two parts. The events from the first $2 / 3$ of the interval - approximately 700 events - represent the "teaching set", the rest - about 300 events - make up the "test set". From the "teaching set" we extract two uneven groups of events. The first one consisting of 10 successive time events preceding each of the earthquake $(m>6)$ is shown in green in Fig. 8. The rest of the events represent the second (pink) cluster from Fig. 8. The clusters are visualized by using multi-dimensional scaling in 3-D. Then, the distances (see Eqs. 3-4) between events belonging to the same clusters was multiplied by the factor $\lambda<1$, while the distances between events from different clusters remain the same. The value of $\lambda$ is gradually decreasing to the moment when the two clusters separates from each other. For the situations shown in Fig. $8 \lambda$ is set to 0.8 . The events from the "test set" (the blue points) are added to this "teaching" structure, with the determined coordinates in 3-D $\omega$ space. They are "attracted" or "repelled" from the "teaching" clusters according to their distances to the events from the "teaching set". Eventually, we obtain the configurations shown in Fig. 8. The precursory events from the test set marked in blue (10 events preceding the earthquake) were recognized at $100 \%$ level for synthetic data sets A, U, F. The blue points fall into the area occupied by the green cluster representing precursory events from the "teaching set". They can be classified by using simple k-NN (k nearest neighbors) classifier. We see that many other points are also situated in the area of a green cluster. However, the choice of precursory events for training was completely arbitrary. By using a more careful analysis of seismicity patterns, such as in (Eneva and Ben-Zion, 1997a), and a better selection of "teaching" patterns, we can improve the classification.

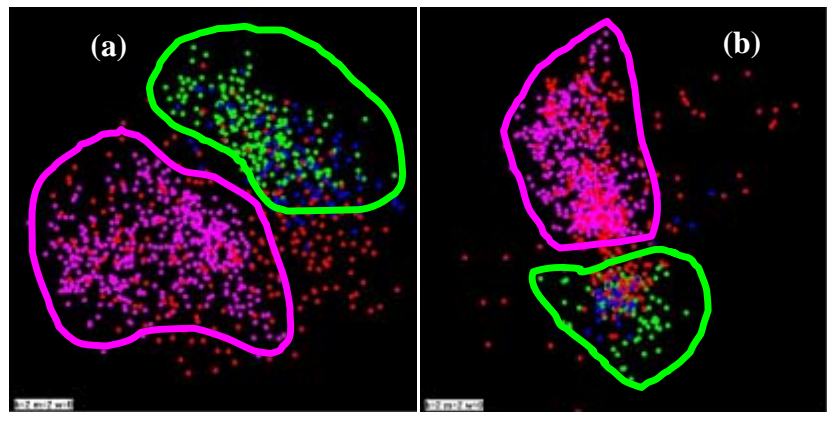

Fig. 8. Results from visual classification of the synthetic data (from catalogs A and F, respectively) in the feature space transformed into 3-D space by using multi-dimensional scaling (MDS). The time events are divided onto two groups of data $\left(F_{i} ; i<T\right)$ (violet and green) and $\left(F_{i} ; i>T\right)$ (red and blue) representing: teaching and test sets, respectively. The classifier is constructed using teaching set of time events, which consists of two groups of events: preceding the earthquakes (green) and the others (violet). The preceding time events were taken arbitrarily as the 10 nearest events to the corresponding earthquake. In the result of training we obtain two clusters: green and violet. The same two groups of data are marked in the test set (preceding the earthquakes - blue, and the others - red). We see that all the blue points were attracted to the green cluster - taught initially as the cluster consisting of "precursory" events so all of them can be recognized as "precursory" (e.g. using k-NN classifier). This is visualized using the authors' own package and the borders of clusters are drawn manually.

\section{Conclusions}

The problem of earthquake prediction, based on data extraction of precursory phenomena, is a highly challenging task. Various computational methods and tools are used for detection of precursors by extracting general information from noisy data. In our opinion, such a generalization is impossible when the data analysis is carried out at a single level of resolution. Improved possibilities exist when using a set of data-mining tools interactively, allowing for on-line clustering, feature extraction and visualization of the data on various levels of resolution.

We show that by using a common framework of clustering, we are able to perform multi-resolutional analysis of seismic data starting from the raw data events described only by their magnitude-spatio-temporal data space. Then we look for global cluster structure in the feature space, which is defined by using the seismicity parameters. This global view can also be divided over different levels of resolution in the feature space defined, e.g. by wavelet analysis (Holschneider, 1995; Strang and Nguyen, 1996) of the time series of the seismicity parameters (Torrence and Compo, 1998) and further classification of wavelet amplitudes by using clustering schemes.

Our present approach is different from that presented earlier (Dzwinel et al., 2003). The fine-grained spatio-temporal patterns of correlated events, extracted by using agglomerative clustering schemes, can be analyzed further in the coarse 
grained feature space by eliminating the noisy patterns and uncorrelated events. We have developed a new software that is based on pre-clustering. This allows for the detection of precursory events with a higher accuracy (e.g. pick-up the Miyakejima event) and their generalization at the low resolution level. It also allows us to construct visual classifiers for anonymous data. We believe that a more careful extraction of the precursory events will be needed for constructing more accurate classifiers.

The raw seismic data contain both local and global knowledge of the correlations between the seismic events. Thus, the two-level approach presented here can still be incomplete, since the general knowledge about the seismic background can be buried in the subtle patterns of data events. Any coarse graining of the data can destroy some, if not a majority, of these patterns. Therefore, extracting global knowledge about seismic patterns corresponding to precursory events involves global clustering of data without any averaging. This is a very daunting task both methodologically and computationally. In particular, great difficulties are associated with the irregular structure of seismic data, which comprise many noisy events, different accuracy of measurements, outliers, bridges, clusters of different density and the large number of data vectors (greater than $10^{4}$ ) which have to be processed. This problem can be attacked by using modern non-hierarchical clustering schemes, such as the DBSCAN (Sander et al., 1998), CURE (Guha and Rastogi, 1998), CHAMELEON (Karypis, 1999) or the shared nearest neighbor clustering algorithm (SNNCA) (Ertoz, 2003).

This new methodology can be also used for the analysis of the data from other geological phenomena, e.g, we can apply this clustering method to volcanic eruptions (Amelung et al., 2000; Hong et al., 2004), astrophysical events such as dissipation phenomena, occurring in a dispersed stellar population (Briceno et al., 2001) also in data mining and bioinformatics (Kuramochi and Karypis, 2001; Jones and Pevzner, 2004).

\section{Glossary}

- features denoted $f_{i}$ or $F_{j}(i, j$ - feature indices) - a set of variables which carry discriminating and characterizing information about the objects under consideration. The features can represent raw measurements (data) $f_{i}$ or can be generated in a non-linear way from the data $F_{j}$ (features).

- data vector ( $f_{k}$ where $k$ - data vector index) and feature vector $\left(\boldsymbol{F}_{k}\right.$ where $l-$ feature vector index $)$ - a collection of features ordered in some meaningful way into multi-dimensional column vectors $\boldsymbol{f}_{k}$ and $\boldsymbol{F}_{l}$ that represents the signature of the object to be identified represented by raw data $f_{i}$ or generated features $F_{j}$. The dimensionalities of data and feature vectors can be different.
- data space - the multidimensional space in which the data vectors $\boldsymbol{f}_{k}$ exist.

- feature space - the multidimensional space in which the $\boldsymbol{F}_{k}$ vectors are defined. Data and feature vectors represent multidimensional points in respective spaces.

- class - the category to which a given object belongs. The classes can create patterns in a properly defined multidimensional space.

- cluster - isolated set of multidimensional points (objects) in data and feature spaces.

- clustering - the computational procedure extracting clusters in multidimensional spaces.

- agglomerative clustering algorithm - the algorithm of a clustering procedure in which the clusters are builtup in a hierarchical way. At the start of clustering, the multidimensional points represent singular clusters. The procedure repeats the process of gluing-up the closest clusters to the moment when a proper number of clusters is achieved.

- non-hierarchical clustering algorithm - the clustering algorithm in which the clusters are searched for by using global optimization algorithms (unlike in agglomerative clustering where local search is exploited). The most representative algorithms of this type are constructed on the base of k-means procedure.

- k-means clustering (Theodoris and Koutroumbas, 1998) - non-hierarchical clustering algorithm in which the randomly generated centers of clusters are then improved iteratively. This simple idea is exploited in various variants of this algorithm.

- multi-resolutional clustering analysis - due to clustering a hierarchy of clusters can be obtained. The analysis of the results of clustering in various resolution levels allows for extraction of knowledge hidden in both local (small clusters) and global (large clusters) similarity of multidimensional points (objects).

- data mining (Mitra and Acharya, 2003) - algorithms, tools, methods and systems used in extraction of knowledge hidden in a large amount of data.

Acknowledgements. We acknowledge the Japan Meteorological Agency (JMA), which allowed us to use their hypocentral data set We thank George Karypis, Y. J. B. D. Kaneko Zack, A. Garbow, B. J. Kadlec, E. Bolling and G. Erlebacher for useful discussions and $\mathrm{K}$. Tiampo for valuable comments and remarks. WD and KB acknowledge support from the Polish Committee for Scientific Research (KBN) Grant No. 3T11C05926. DAY acknowledges support from both the Geophysics and the ITR programs. YBZ acknowledges support from a Mercator fellowship of the German Research Society (DFG).

Edited by: G. Zöller

Reviewed by: S. Abe, K. F. Tiampo and another referee 


\section{References}

Amelung, F., Jonsson, S., Zebker, H., and Segall, P.: Widespread uplift and "trapdoor" faulting on Galapagos volcanoes observed with radar interferometry, Nature, 407, 993-996, 2000.

Andenberg, M. R.: Clusters Analysis for Applications, New York, Academic Press, 1973.

Anghel, M., Ben-Zion Y., and Martinez, R. R. : Dynamical system analysis and forecasting of deformation produced by an earthquake fault, Pure Appl. Geophys., 161, 2023-2051, 2004.

Ben-Zion, Y. and Rice, J. R.: Slip patterns and earthquake populations along different classes of faults in elastic solids, J. Geophys. Res., 100, 12, 12 959-12983, 1995.

Ben-Zion, Y.: Stress, slip and earthquakes in models of complex single-fault systems incorporating brittle and creep deformations, J. Geophys. Res., 101, 5677-5706, 1996.

Ben-Zion, Y. and Lyakhovsky, V.: Accelerated seismic release and related aspects of seismicity patterns on earthquake faults, Pure Appl. Geophys., 159, 2385-2412, 2002.

Boryczko, K., Dzwinel, W., and Yuen, D. A.: Clustering Revealed in High-Resolution Simulations and Visualization of MultiResolution Features in Fluid-Particle Models, Concurr. CompPract., 15, 101-116, 2003.

Briceno, C., Vivas, A. K., Calvet, N., Hartmann, L., Pacheco, R., Herrera, D., Romero, L., Berlind, P., Sanchez, G., Snyder, J. A., and Andrews, P.: The CIDA-QUEST Large-Scale Survey of Orion OB1: Evidence for Rapid Disk Dissipation in a Dispersed Stellar Population, Science, 291, 93-96, 2001.

Dowla, F. U.: Neural networks in seismic discrimination, in Monitoring a CTBT, edited by Husebye E. S. and Dainty, A. S., NATO-ASI series E 303, Kluwer Publishing, 777-789, 1995.

Dzwinel, W.: How to Make Sammon's Mapping Useful for Multidimensional Data Structures Analysis?, Pattern Recogn., 27/7, 949-959, 1994.

Dzwinel W. and Blasiak, J.: Method of particles in visual clustering of multi-dimensional and large data sets, Future Gener. Comp. S., 15, 365-379, 1999.

Dzwinel, W., Yuen, D. A., Kaneko, Y., Boryczko, K., and Ben-Zion, Y.: Multi-Resolution Clustering Analysis and 3-D Visualization of Multitudinous Synthetic Earthquakes, Vis. Geosc., 8, 12-25, 2003.

Eneva, M. and Ben-Zion, Y.: Techniques and parameters to analyze seismicity patterns associated with large earthquakes, J. Geophys. Res., 102, 177 85-17 795, 1997a.

Eneva, M. and Ben-Zion, Y.: Application of pattern recognition techniques to earthquake catalogs generated by models of segmented fault systems in three-dimensional elastic solids, J. Geophys. Res., 102, 24 513-24 528, 1997 b.

Enescu, B., Ito, K., and Struzik, Z.: (Multi)fractality of Earthquakes by use of Wavelet Analysis. Proceedings of American Geophysical Union Fall Meeting, 6-10 December 2002, San Francisco, California, 2002.

Erlebacher, G. and Yuen, D. A.: A Wavelet Toolkit for Visualization and Analysis of Large Data Sets In Earthquake Research, Pure Appl. Geophys., 161, 2215-2229, 2004.

Erlebacher, G., Yuen, D. A., and Dubuffet, F. W.: Current trends and demands in visualization in the geosciences, http://link.springer-ny.com/link/service/journals/10069/technic/ erlebach/index.htm, Electr. Geosc., 4, 2001.

Ertoz, L., Steinbach, M., and Kumar, V.: Finding Clusters of Different Size, Shapes and Densities in Noisy, High-Dimensional Data, Army High Performance Center, technical report, April 2003.
Freed A. M. and Lin, J.: Delayed triggering of the $1999 \mathrm{Hec}-$ tor Mine earthquake by viscoelastic stress transfer, Nature, 411, 180-183, 2001.

Geller, R. J., Jackson, D. D., Kagan, Y. Y., and Mulargia, Y. F.: Earthquakes Cannot Be Predicted, Science Online, 275, 5306, 1616, 1997.

Gowda, C. K. and Krishna, G.: Agglomerative clustering using the concept of nearest neighborhood, Pattern Recogn., 10, 105-112, 1978.

Grossman, R. L., Karnath, Ch., Kegelmeyer, P., Kumar, V., and Namburu, R. R.: Data Mining for Scientific and Engineering Applications, Kluwer Academic Publisher, New York, 2001.

Guha, S., Rastogi, R., and Cure, A.: An efficient clustering algorithm for large data bases, SIGMOD 1998, Proceedings of ACM SIGMOD International Conference on Management of Data, Washington, USA, 73-84, 1998.

Gutenberg, B. and Richter, C. F.: Frequency of earthquakes in California, Bul. Seismol. Sco. Amer., 34, 185-188, 1944.

Hand, D., Mannila, H., and Smyth, P.: Principles of Data Mining, M.I.T. Press, Cambridge, MA, 2001.

Hastie, T., Tibshirani, R., and Friedman, J.: The Elements of Statistical Learning: Data Mining, Inference and Prediction, Springer Verlag, New York, 533, 2001.

Haile, P. M.: Molecular Dynamics Simulation, Wiley: New York, 1992.

Holschneider, M.: Wavelets: An Analysis Tool, Oxford Science Publications, Clarendon Press, Oxford, 1995.

Hong, H., Kadlec, D. J., Yuen, D. A., Zheng, Y., Zhang, H., Liu, G., and Dzwinel, W.: Fast timescale phenomena at Changbaishan volcano as inferred from recent seismic activity, Eos Trans. AGU, Fall Meet., 85, 47, 2004.

Ito, T. and Yoshioka, S.: A dike intrusion model in and around Miyakejima, Niijima and Kozushima, Tectonophysics, 359, 171187, 2002.

Ismail, M. A. and Kamel, M. S.: Multidimensional data clustering utilizing hybrid search strategies, Pattern Recogn., 22, 1, 77-89, 1989.

Jain, D. Dubes, R. C.: Algorithms for Clustering Data, PrenticeHall Advanced Reference Series, 1988.

Joswig, M.: Pattern recognition for earthquake detection, Bull. Seism. Soc. Am., 80, 170-186, 1990.

Jones, N. C. and Pevzner, P. A.: An Introduction to Bioinformatics Algorithms, MIT Press, Cambridge MA, 435, 2004.

Karypis, G., Han, E., and Kumar, V.: Chameleon: A hierarchical clustering algorithms using dynamic modeling, IEEE Computer, 32, 8, 68-75, 1999.

Karypis, G. and Kumar, V.: Multilevel algorithms for multiconstraint graph partitioning, technical report, University of Minnesota Supercomputing Institute, Minneapolis, MN, 1998.

Keilis-Borok, V. I. and Kossobokov, V. G.: Premonitory activation of earthquake flow: Algorithm M8, Phys. Earth Planet. Inter., 61, 73-83, 1990.

Kuramochi, M. and Karypis, G.: Gene Classification using Expression Profiles: A Feasibility Study, Proceedings of 2nd IEEE Symposium on Bioinformatics and Bioengineering, 4-6 November 2001, Rockville, MD, 2001.

Miller, S. A., Ben-Zion, Y., and Burg, J. P.: A three-dimensional fluid-controlled earthquake model: behavior and implications, J. Geoph. Res., 104/B5, 10 621-10 638, 1999.

Mitra, S. and Acharya, T.: Data Mining: Multimedia, Soft Computing and Bioinformatics, J. Wiley, 424, 2003.

Rundle, J. B., Gross, S., Klein, W., Ferguson, C., and Turcotte, 
D. L.:The statistical mechanics of earthquakes, Tectonophysics, 277, 147-164, 1997.

Rundle, J. B., Klein, W., Tiampo, K., and Gross, S.: Linear pattern dynamics in nonlinear threshold systems, Phys. Rev. E, 61, 3, 2418-2143, 2000a.

Rundle, J. B., Turcotte, D. L., and Klein, W. (eds.): GeoComplexity and the Physics of Earthquakes, AGU, Washington D.C., 284, 2000b.

Rundle, J. B., Tiampo, K. F., Klein, W., and Martins J. S. S.: Selforganization in leaky threshold systems: The influence of nearmean field dynamics and its implications for earthquakes, neurobiology, and forecasting, Proc. Nat. Acad. Sci, 99 suppl., 25142521, 2002.

Sander, J., Ester, M., and Krieger, H.: Density based clustering in spatial databases, The algorithm DBSCAN and its applications, Data Mining and Knowledge Discovery, 2, 2, 169-194, 1998.

Siedlecki, W., Siedlecka, K., and Sklanski, J.: An overview of mapping for exploratory pattern analysis, Pattern Recogn., 21, 5, 411-430, 1988.

Song, T. A. and Simons, M.: Large trench-parallel gravity variations predict seismogenic behavior in subduction zones, Science, 301, 630-633, 2003.

Strang, G. and Nguyen, T.: Wavelets and Filter Banks, WellesleyCambridge Press, Wellesley, MA, 1996.

Theodoris, S. and Koutroumbas K.: Pattern Recogn., Academic Press, San Diego, London, Boston, 1998.

Tiampo, K. F., Rundle, J. B., Gross, S. J., McGinnis, S., Gross, S. J., and Klein, W.: Eigen patterns in southern California seismicity, J. Geophys. Res. - Solid Earth, 107, B12, 2354, doi:10.1029/2001JB000562, 2002a.
Tiampo, K. F., Rundle, J. B., McGinnis, S., Gross, S. J., and Klein, W.: Mean field threshold systems and phase dynamics: An application to earthquake fault systems, Europhys. Lett., 60, 481-487, 2002b.

Tiira, T.: Detecting teleseismic events using artificial neural networks, Comput. Geosci-UK, 25, 929-939, 1999.

Toda, S., Stein, R. S., and Sagiya, T.: Evidence from the AD 2000 Izu Islands earthquake swarm that stressing rate governs seismicity, Nature, 419, 58-61, 2002.

Wang, W. and Gengfeng, W.: A New Generation Expert System for Earthquake Prediction based on Fuzzy Neural Networks and Symbols, Selected Papers on Earthquake Precursor and Prediction in China, Seismological Press, 222-231, 1996.

Wesnousky, S. G.: The Gutenberg-Richter or characteristic earthquake distribution, which is it?, Bull. Seismol. Soc. Amer., 84, 1940-1959, 1994.

Wiemer, S. and Wyss, M.: Mapping spatial variability of the frequency-magnitude distribution of earthquakes, Adv. Geophys., 45, 259-302, 2002.

Xiaowei, Xu., Jager, J., and Kriegel, H-P.: A fast parallel clustering algorithm for large spatial databases, Data Mining and Knowledge Discovery, 3, 3, 263-290, 1999.

Zhang, Q. and Boyle, R.: A new clustering algorithm with multiple runs of iterative procedures, Pattern Recogn., 24, 9, 835-848, 1991. 